University of Nebraska - Lincoln

DigitalCommons@University of Nebraska - Lincoln

Faculty Publications from the Department of Engineering Mechanics

2009

\title{
The Magnetoelectric Effects in Multiferroic Composite Nanofibers
}

C. L. Zhang

Zhejiang University, Hangzhou, 310027 Zhejiang, People's Republic of China

W. Q. Chen

Zhejiang University, Hangzhou, 310027 Zhejiang, People's Republic of China

S. H. Xie

Zhejiang University, Hangzhou, 310027 Zhejiang, People's Republic of China

J. S. Yang

University of Nebraska-Lincoln, jyang1@unl.edu

Jiangyu Li

University of Washington, jjli@uw.edu

Follow this and additional works at: https://digitalcommons.unl.edu/engineeringmechanicsfacpub

Part of the Mechanical Engineering Commons

Zhang, C. L.; Chen, W. Q.; Xie, S. H.; Yang, J. S.; and Li, Jiangyu, "The Magnetoelectric Effects in Multiferroic Composite Nanofibers" (2009). Faculty Publications from the Department of Engineering Mechanics. 40.

https://digitalcommons.unl.edu/engineeringmechanicsfacpub/40

This Article is brought to you for free and open access by the Mechanical \& Materials Engineering, Department of at DigitalCommons@University of Nebraska - Lincoln. It has been accepted for inclusion in Faculty Publications from the Department of Engineering Mechanics by an authorized administrator of DigitalCommons@University of Nebraska - Lincoln. 


\title{
The magnetoelectric effects in multiferroic composite nanofibers
}

\author{
C. L. Zhang, ${ }^{1}$ W. Q. Chen, ${ }^{1}$ S. H. Xie, ${ }^{2}$ J. S. Yang, ${ }^{3}$ and J. Y. Li $\left.{ }^{4}, \mathrm{a}\right)$ \\ ${ }^{1}$ Department of Civil Engineering, Zhejiang University, Hangzhou, 310027 Zhejiang, \\ People's Republic of China \\ ${ }^{2}$ Key Laboratory of Low Dimensional Materials and Application Technology of Ministry of Education, \\ and Faculty of Materials, Optoelectronics and Physics, Xiangtan University, Xiangtan, 411105 Hunan, \\ People's Republic of China \\ ${ }^{3}$ Department of Engineering Mechanics, University of Nebraska, Lincoln, Nebraska 68588, USA \\ ${ }^{4}$ Department of Mechanical Engineering, University of Washington, Seattle, Washington 98195-2600, USA
}

(Received 12 January 2009; accepted 15 February 2009; published online 13 March 2009)

In this letter, we analyze the quasistatic and dynamic magnetoelectric responses of multiferroic composite nanofibers consisting of both ferroelectric and ferromagnetic phases and demonstrate that the nanofibers exhibit magnetoelectric responses orders of magnitude higher than multiferroic composite thin films of similar compositions. The analysis suggests that the multiferroic nanofibers are promising for magnetoelectric applications. (C) 2009 American Institute of Physics.

[DOI: $10.1063 / 1.3095596]$

Magnetoelectric coupling in multiferroic materials has attracted great interests in recent years not only for their fundamental scientific significance but also for their potential applications in sensing, actuation, data storage, and spintronics. ${ }^{1-4}$ Since magnetoelectric effect in single-phase multiferroics is rather rare and small and often exists only at low temperature, ${ }^{5}$ hybrid multiferroic composites consisting of ferroelectric and ferromagnetic phases have been widely explored, ${ }^{6}$ where room temperature magnetoelectric coupling not existing in either constituent is induced in the composites through the mechanical interactions between piezoelectric and magnetostrictive effects. ${ }^{7-10}$ Many of the studies have focused on low-dimensional thin films of multiferroic composites, ${ }^{3,11-13}$ yet the mechanical constraint of film substrate has severely limited their magnetoelectric response. Multiferroic nanofibers have been proposed as an alternative to magnetoelectric thin films and have recently been synthesized by sol-gel based electrospinning. ${ }^{14-16}$ In this letter, we show from simple analysis that the multiferroic composite nanofibers indeed possess magnetoelectric coupling that is orders of magnitude higher than multiferroic composite thin films of similar compositions, and thus are promising for practical applications.

To be specific, we consider a multiferroic composite nanofiber consisting of both ferroelectric and ferromagnetic phases and is aligned and electrically poled along $x_{1}$ axis. As such, the constitutive equations of the nanofibers are given by

$$
\begin{aligned}
& \varepsilon_{1}=S_{11} \sigma_{1}+d_{k 1} E_{k}+q_{k 1} H_{k}, \\
& D_{i}=d_{i 1} \sigma_{1}+\kappa_{i k} E_{k}, \\
& B_{i}=q_{i 1} \sigma_{1}+\mu_{i k} H_{k},
\end{aligned}
$$

where $\varepsilon_{1}, D_{i}$, and $B_{i}$ are strain, electric displacement, and magnetic induction, respectively, $\sigma_{1}, E_{k}$, and $H_{k}$ are stress, electric field, and magnetic field, respectively, and $S_{11}, \kappa_{i k}$, and $\mu_{i k}$ are elastic compliance, dielectric permittivity, and

\footnotetext{
a) Author to whom correspondence should be addressed. Electronic mail: jjli@u.washington.edu.
}

magnetic permeability, respectively. Due to the onedimensional nature of nanofiber, we only have nonzero stress component in $x_{1}$ direction. The nanofiber exhibits both piezoelectric effect from its ferroelectric phase and magnetostrictive effect from its ferromagnetic phase, as demonstrated by the piezoelectric coefficient $d_{k 1}$ and piezomagnetic coefficient $q_{k 1}$. However, it does not exhibit magnetoelectric effect at the material level, as evident from its lack of magnetoelectric coefficient connecting electric and magnetic field variables in the constitutive equations.

Now consider such a nanofiber as a beam structure with both ends fixed, and thus any extensional deformation in the nanofiber is prohibited. When a magnetic field $H_{k}$ either along or perpendicular to the fiber is applied, the strain due to the magnetostrictive effect is blocked due to the mechanical constraint at both ends, and stress is induced in the fiber as a result, which can be solved as

$$
\sigma_{1}=\widetilde{C}_{11} \varepsilon_{1}-\widetilde{e}_{k 1} E_{k}-\tilde{h}_{k 1} H_{k}=-\tilde{e}_{k 1} E_{k}-\tilde{h}_{k 1} H_{k},
$$

with

$$
\tilde{C}_{11}=\frac{1}{S_{11}}, \quad \tilde{e}_{k 1}=\frac{d_{k 1}}{S_{11}}, \quad \tilde{h}_{k 1}=\frac{q_{k 1}}{S_{11}} .
$$

The stress will then induce electric response in the fiber due to its piezoelectric effect. By substituting Eq. (4) into Eq. (2), we obtain the electric displacement as

$$
D_{i}=\tilde{e}_{i 1} \varepsilon_{1}+\tilde{\kappa}_{i k} E_{k}+\tilde{a}_{i k} H_{k}=\tilde{\kappa}_{i k} E_{k}+\tilde{a}_{i k} H_{k},
$$

with

$$
\tilde{\kappa}_{i k}=\kappa_{i k}-\frac{d_{i 1} d_{k 1}}{S_{11}}, \quad \tilde{a}_{i k}=-\frac{d_{i 1} q_{k 1}}{S_{11}} .
$$

It is noted that the magnetoelectric coupling is now induced in the nanofiber at the structure level, as demonstrated by the magnetoelectric coefficient $\tilde{a}_{i k}$. Under the open-circuit condition where $D_{1}=0$, we can solve Eq. (6) for the induced $E_{1}$ in term of the applied magnetic field $H_{k}$. A dimensionless parameter $r_{1 k}$ is introduced to characterize the magnetoelectric response of the nanofiber, 


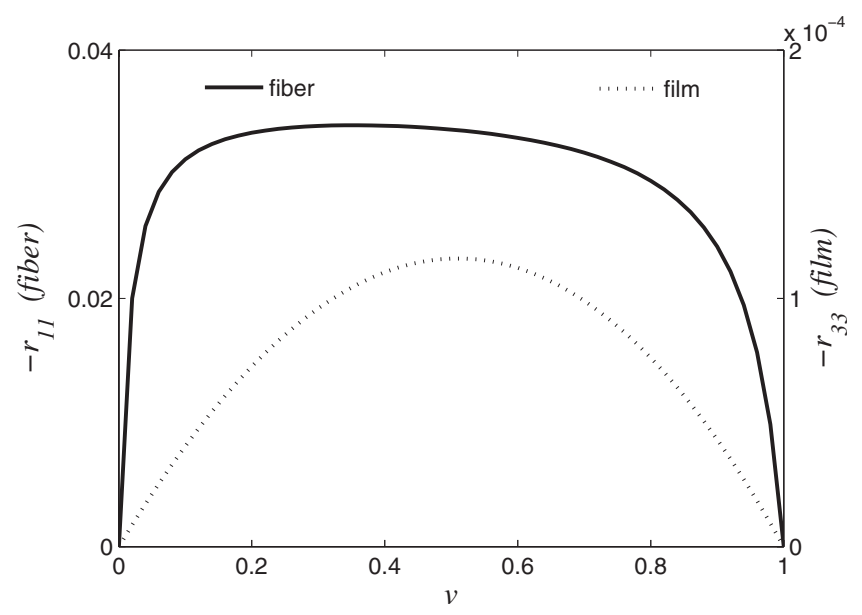

FIG. 1. The static longitudinal magnetoelectric coefficient of multiferroic composite nanofiber and thin film vs the volume fraction of PZT.

$$
r_{1 k}=\frac{\sqrt{\epsilon_{0}}}{\sqrt{\mu_{0}}} \frac{E_{1}}{H_{k}}=-\frac{\sqrt{\epsilon_{0}}}{\sqrt{\mu_{0}}} \frac{\tilde{a}_{1 k}}{\widetilde{\kappa}_{11}}=\frac{\sqrt{\epsilon_{0}}}{\sqrt{\mu_{0}}} \frac{d_{11} q_{k 1}}{\kappa_{11} S_{11}-d_{11}^{2}},
$$

with the longitudinal response due to $H_{1}$ characterized by $r_{11}$ and transverse response due to $H_{3}$ by $r_{13}$. Note that both $\widetilde{\kappa}_{12}$ and $\widetilde{\kappa}_{13}$ vanish due to transverse isotropic symmetry of poled ferroelectrics.

To compare the magnetoelectric response of nanofibers with thin films of similar compositions, we plot the longitudinal magnetoelectric coefficient $r_{11}$ of nanofiber and $r_{33}$ of thin film, as shown in Fig. 1. Both the nanofiber and thin film consist of ferroelectric lead zirconate titanate and ferromagnetic $\mathrm{CoFe}_{2} \mathrm{O}_{4}$, and the thin film composite has bilayered structure on a thick substrate, with film thickness along $x_{3}$ axis. The detailed analysis of the bilayered multiferroic composites can be found in Zhang et al. ${ }^{17}$ and will not be repeated here. PZT has $d_{11}=289 \times 10^{-12} \mathrm{C} / \mathrm{N}, S_{11}=15.5$ $\times 10^{-12} \mathrm{~m}^{2} / \mathrm{N}$, and $\kappa_{11}=5.52 \times 10^{-9} \mathrm{C}^{2} \mathrm{~N} / \mathrm{m}^{2}$, while $\mathrm{CoFe}_{2} \mathrm{O}_{4}$ has $q_{11}=1.88 \times 10^{-9} \mathrm{~m} / \mathrm{A}, q_{31}=5.7 \times 10^{-12} \mathrm{~m} / \mathrm{A}$, and $S_{11}=7 \times 10^{-12} \mathrm{~m}^{2} / \mathrm{N}$ when magnetized along $x_{1}$ direction, and $S_{11}=6.48 \times 10^{-12} \mathrm{~m}^{2} / \mathrm{N}$ when magnetized along $x_{3}$ direction. $^{18,19}$ The material constants of the composite are estimated using a simple volume averaging. It is observed that nanofiber exhibits highest magnetoelectric response at $50 \%$ of PZT and has magnetoelectric coefficient that is two orders of magnitude higher than that of thin film, suggesting that the multiferroic nanofibers are indeed promising for magnetoelectric applications. Similar observations can also be made on transverse response, as shown in Fig. 2. It is also noted that the transverse magnetoelectric response of nanofiber is smaller than its longitudinal one, while the trend for thin film is reversed.

It is well known that the mechanical resonance of the composite structure can be used to enhance the magnetoelectric response of the composite substantially. ${ }^{20}$ To investigate this dynamic effect, we consider a harmonic magnetic field of magnitude $A_{k}$ and frequency $\omega$, as given by $H_{k}$ $=A_{k} \exp (i \omega t)$. Under the steady state condition, all the field variables have the same time dependence, and thus will be dropped for convenience. The motion equation and the electric equilibrium equation of the nanofiber then become

$$
\sigma_{1,1}=\widetilde{C}_{11} u_{1,11}+\widetilde{e}_{11} \phi_{, 11}=-\rho \omega^{2} u_{1}
$$

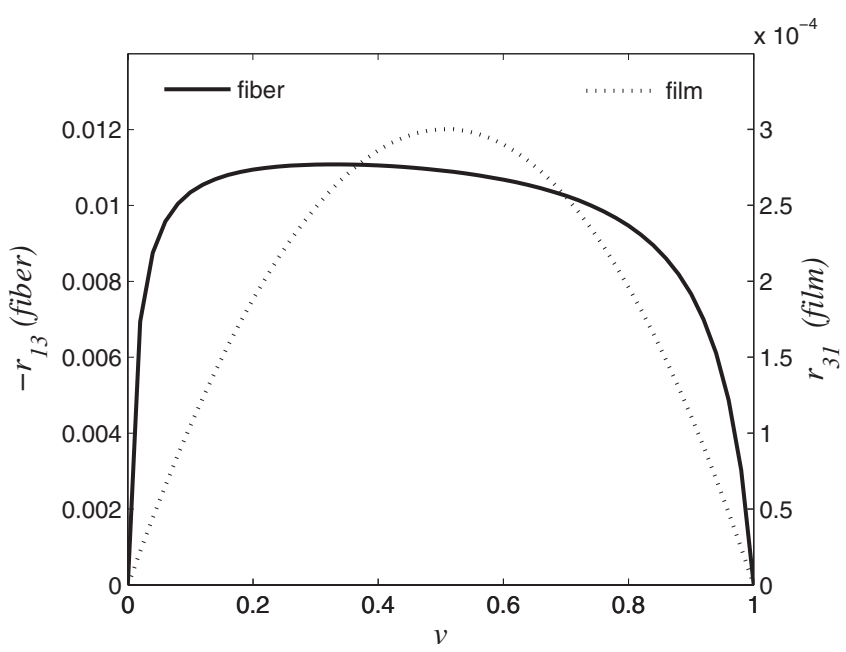

FIG. 2. The static transverse magnetoelectric coefficient of multiferroic composite nanofiber and thin film vs the volume fraction of PZT.

$$
D_{1,1}=\widetilde{e}_{11} u_{1,11}-\widetilde{\kappa}_{11} \phi_{, 11}=0,
$$

in which the gradient of the uniform magnetic field applied vanishes. The equations can be solved for the induced electric field under free-end mechanical boundary condition as

$$
\begin{aligned}
E_{1} & =-\left[\frac{\tilde{h}_{k 1}-\frac{\tilde{e}_{11}}{\tilde{\kappa}_{11}} \tilde{a}_{1 k}}{\left(\tilde{C}_{11} \frac{\tilde{\kappa}_{11}}{\tilde{e}_{11}}+\tilde{e}_{11}\right)} \frac{\cos k x_{1}}{\cos k L}+\frac{\tilde{a}_{1 k}}{\tilde{\kappa}_{11}}\right] A_{k} \\
& =\frac{d_{11} q_{k 1}}{d_{11}^{2}-\kappa_{11} S_{11}}\left(\frac{\cos k x_{1}}{\cos k L}-1\right) A_{k},
\end{aligned}
$$

with

$$
k^{2}=\frac{\rho \omega^{2}}{\widetilde{C}_{11}+\frac{\widetilde{e}_{11}^{2}}{\widetilde{\kappa}_{11}},}
$$

which clearly show a maximum magnetoelectric response near mechanical resonance when $\cos k L=0$. The corresponding magnetoelectric coefficient is then given as

$$
r_{1 k}=\frac{\sqrt{\epsilon_{0}}}{\sqrt{\mu_{0}}} \frac{E_{1}}{A_{k}}=\frac{\sqrt{\epsilon_{0}}}{\sqrt{\mu_{0}}} \frac{d_{11} q_{k 1}}{d_{11}^{2}-\kappa_{11} S_{11}}\left(\frac{\cos k x_{1}}{\cos k L}-1\right) .
$$

The transverse dynamic responses of the multiferroic composite nanofiber and thin film as a function of magnetic driving frequency are shown in Fig. 3, with the volume fraction of PZT set to be $25 \%$. To avoid singularity at the resonance, we introduce mechanical damping in the composite, by setting its loss tangent to be $5 \%$. A slight change in the loss tangent, while affecting the absolute value of dynamic magnetoelectric coefficients, does not change the following qualitative comparisons and conclusions. The maximum magnetoelectric responses are indeed observed in both nanofiber and thin film at their mechanical resonance, which are almost two orders of magnitude higher than the corresponding static responses, consistent with experimental observations in bulk composite structures. ${ }^{20}$ In addition, the dynamic magnetoelectric response of nanofiber is also more than one order of magnitude higher than that of thin film, similar to what we observed for static response. Similar ob- 


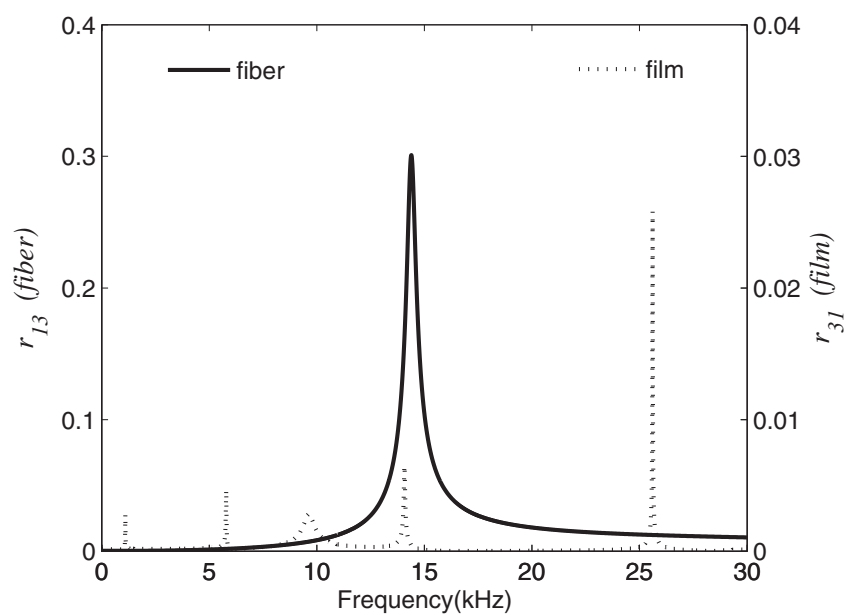

FIG. 3. The dynamic transverse magnetoelectric coefficient of multiferroic composite nanofiber and thin film vs the magnetic driving frequency.

servations can also be made on the longitudinal dynamic magnetoelectric response of nanofiber, as shown in Fig. 4, where the two-dimensional mapping of longitudinal dynamic magnetoelectric coefficient of the nanofiber is plotted as a function of volume fraction of PZT and magnetic driving

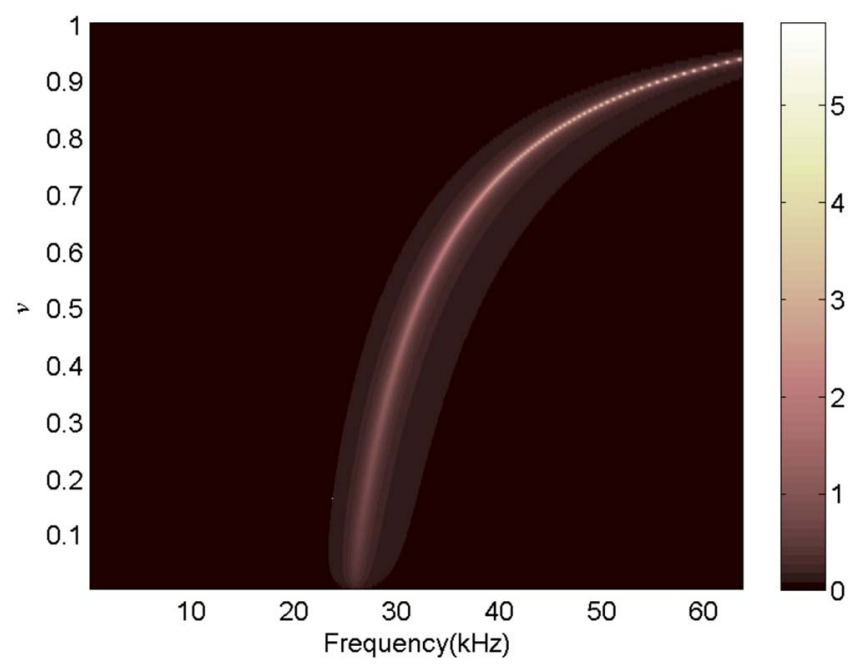

FIG. 4. (Color online) The dynamic longitudinal magnetoelectric coefficient of multiferroic composite nanofiber vs the volume fraction of PZT and the magnetic driving frequency. frequency, from which the optimal volume fraction and driving frequency can be identified.

In conclusion, we show from simple analysis that the multiferroic composite nanofibers have magnetoelectric responses that are orders of magnitude higher than composite thin films of similar compositions, suggesting that multiferroic composite nanofibers are promising for magnetoelectric applications.

The work was partially supported by the National Natural Science Foundation of China (Grant Nos. 10725210, 10832009, 10732100, and 10772155), the Specialized Research Fund for the Doctoral Program of Higher Education (Grant No. 20060335107), and National Science Foundation (Grant Nos. DMR 0706100 and OISE-0820583).

${ }^{1}$ M. Fiebig, J. Phys. D 38, R123 (2005).

${ }^{2}$ N. A. Spaldin and M. Fiebig, Science 309, 391 (2005).

${ }^{3}$ R. Ramesh and N. A. Spaldin, Nature Mater. 6, 21 (2007).

${ }^{4}$ J. R. Hattrick-Simpers, L. Y. Dai, M. Wuttig, I. Takeuchi, and E. Quandt, Rev. Sci. Instrum. 78, 106103 (2007).

${ }^{5}$ W. Eerenstein, N. D. Mathur, and J. F. Scott, Nature (London) 442, 759 (2006).

${ }^{6}$ C. W. Nan, M. I. Bichurin, S. X. Dong, D. Viehland, and G. Srinivasan, J. Appl. Phys. 103, 031101 (2008).

${ }^{7}$ G. Srinivasan, E. T. Rasmussen, J. Gallegos, R. Srinivasan, Y. I. Bokhan, and V. M. Laletin, Phys. Rev. B 64, 214408 (2001).

${ }^{8}$ M. I. Bichurin, V. M. Petrov, and G. Srinivasan, Ferroelectrics 280, 331 (2002).

${ }^{9}$ S. X. Dong, J. F. Li, and D. Viehland, Appl. Phys. Lett. 83, 2265 (2003).

${ }^{10}$ S. Srinivas and J. Y. Li, Acta Mater. 53, 4135 (2005).

${ }^{11}$ H. Zheng, J. Wang, S. E. Lofland, Z. Ma, L. Mohaddes-Ardabili, T. Zhao, L. Salamanca-Riba, S. R. Shinde, S. B. Ogale, F. Bai, Y. Jia, D. Viehland, D. G. Schlom, M. Wuttig, A. Roytburd, and R. Ramesh, Science 303, 661 (2004).

${ }^{12}$ C. W. Nan, G. Liu, Y. Liu, and H. Chen, Phys. Rev. Lett. 94, 197203 (2005).

${ }^{13}$ Z. Y. Li, J. G. Wan, X. W. Wang, Y. Wang, J. S. Zhu, G. H. Wang, and J. M. Liu, Integr. Ferroelectr. 87, 33 (2007).

${ }^{14}$ S. H. Xie, J. Y. Li, Y. Qiao, Y. Y. Liu, L. N. Lan, Y. C. Zhou, and S. T. Tan, Appl. Phys. Lett. 92, 062901 (2008).

${ }^{15}$ S. H. Xie, J. Y. Li, Y. Y. Liu, L. N. Lan, G. Jin, and Y. C. Zhou, J. Appl. Phys. 104, 024115 (2008).

${ }^{16}$ S. H. Xie, J. Y. Li, R. Proksch, Y. M. Liu, Y. C. Zhou, Y. Y. Liu, Y. Ou, L. N. Lan, and Y. Qiao, Appl. Phys. Lett. 93, 222904 (2008).

${ }^{17}$ C. L. Zhang, W. Q. Chen, J. Y. Li, and J. S. Yang, IEEE Trans. Ultrason. Ferroelectr. Freq. Control (unpublished).

${ }^{18}$ S. S. Vel, R. Mewer, and R. Batra, Int. J. Solids Struct. 41, 1625 (2004)

${ }^{19}$ F. Ramirez, P. R. Heyliger, and E. Pan, J. Sound Vib. 229, 1625 (2006).

${ }^{20}$ S. X. Dong, J. F. Li, D. Viehland, J. Cheng, and L. E. Cross, Appl. Phys. Lett. 85, 3534 (2004). 